\title{
VERBUNDTAG 2018 (KLAGENFURT, 15. MAI 2018)
}

von Mathis Kronschläger

Zusammenfassung: Der Verbundtag ist die jährlich stattfindende Tagung zur Diskussion aktueller Themen im Österreichischen Bibliothekenverbund, organisiert von der Österreichischen Bibliothekenverbund und Service GmbH (OBVSG) und einer Partnerbibliothek aus dem Verbund. Der Schwerpunkt des 14. Verbundtags am 15. Mai 2018 an der Universitätsbibliothek Klagenfurt lag auf der Einführung des neuen cloudbasierten Bibliotheksmanagementsystems Alma und der damit einhergehenden Neugestaltung der Verbundarchitektur.

Schlüsselwörter: OBV; Österreichischer Bibliothekenverbund; OBVSG; Universitätsbibliothek Klagenfurt; Tagung; Verbundtag 2018; Alma; Parallelbetrieb; Primo; GND; Zentrale Services; ZDB; Kooperation E-Medien Österreich

\section{ANNUAL LIBRARY NETWORK CONFERENCE (KLAGENFURT, MAY 15, 2018)}

Abstract: The annual library network conference provides the Austrian library community with the opportunity to discuss current topics. The event is organised by the Austrian Library Network and Service Ltd. (OBVSG) and an OBV partner library. The central topic of this year's meeting on May 15 at the University Library Klagenfurt has been the introduction of the new library management system Alma and its impact on the OBV's general architecture.

Keywords: OBV; Austrian Library Network; OBVSG; Austrian Library Network and Service Ltd.; University Library Klagenfurt; conference; Verbundtag 2018; Alma; parallel operations; Primo; GND; central services; ZDB; Austrian Academic Library Consortium

DOI: https://doi.org/10.31263/voebm.v71i2.2141 


\section{Verbundtag 2018 des Österreichischen Bibliothekenverbundes an der Universitätsbibliothek Klagenfurt (15. Mai 2018)}

Bereits zum vierzehnten Mal veranstaltete der OBV seinen jährlichen Verbundtag. Diesmal lud die Alpen-Adria-Universität Klagenfurt auf ihren schönen Campus am Wörthersee.

Rund 90 Expertinnen und Experten aus nahezu allen Verbundbibliotheken trafen sich in offener Atmosphäre, um über Neuigkeiten im Bibliothekswesen, anstehende Herausforderungen im Verbund und gemeinsame Strategien zu beraten.

Der diesjährige Verbundtag stand ganz im Zeichen des Systemwechsels von Aleph auf Alma und dem geglückten Go-Live der Alma Netzwerkzone. Im Plenum am Vormittag bot Wolfgang Hamedinger, Geschäftsführer der OBVSG, einen Gesamtüberblick vom Werdegang des AlmaProjekts im OBV. Daran anschließend berichtete Bettina Kann, ebenfalls OBVSG, über den aktuellen Stand des Aleph-Alma-Parallelbetriebs. Nach einem ausgiebigen Mittagessen hatten die frisch gestärkten Teilnehmerinnen und Teilnehmer am Nachmittag die Möglichkeit, in zwei nacheinander stattfindenden Seminarblöcken je nach Interesse aus mehreren parallel stattfindenden Veranstaltungen zu wählen.

Folgende Themen standen im ersten Block zur Auswahl: die Aleph-Sharing-Bibliotheken und Alma, die Primo-Welt in der neuen Alma-Umgebung und die neu aufgestellte Zentrale Redaktion stellte sich vor. In einem zweiten Block fanden folgende Workshops statt: Alma im OBV (MAB2/MARCKonverter, GND, Schulungsgruppe, Diensteanbindung), das Forum freier Themen mit einem Vortrag zur ZDB-Bestandsdatenlieferung (ein Beitrag zu barrierefreier Literatur musste leider entfallen) und schließlich stellte Ex Libris in einem Workshop seine Produkte Leganto und Esploro vor.

Daneben bestand - mittlerweile schon aus Tradition - in einem eigenen „OBVSG-Aussprachezimmer" für Verbundmitglieder die Möglichkeit, abseits vom Alltagsgeschäft Vorschläge, Ideen sowie Kritik mit der Verbundzentrale zu besprechen.

Zum ersten Mal war beim diesjährigen Verbundtag die Kooperation E-Medien Österreich (KEMÖ) mit einem Infostand vertreten und bot in der Aula der Universität Antworten auf Fragen rund um E-Medien und Open Access.

Beim abschließenden Programmpunkt „Resümee“ waren alle Teilnehmenden wieder versammelt: Kurze Berichte der Moderatorinnen und Moderatoren der Workshops gaben einen Überblick über die Vorträge und Diskussionen des Tages. 
Geselligen Ausklang fand der Verbundtag bei einer anschließenden Führung durch Bibliothek und Campus und einem gemeinsamen Abendessen im Seepark Hotel.

Mag. Mathis Kronschläger Die Österreichische Bibliothekenverbund und Service GmbH (OBVSG) E-Mail: mathis.kronschlaeger@obvsg.at 